\title{
Autoconcepto Físico, Personal, Social Y Académico En Adolescentes Chihuahuenses
}

\author{
Perla Jannet Jurado García, (Dra.) \\ Susana Ivonne Aguirre Vásquez (Dra.) \\ Miguel Conchas Ramírez (M.C.) \\ Fernando Mondaca Fernández (M.C.) \\ Raúl Josué Nájera Longoria (Dr.) \\ José René Blanco Ornelas (Dr.) \\ Autonomous University of Chihuahua, Mexico
}

Doi:10.19044/esj.2018.v14n34p162 URL:http://dx.doi.org/10.19044/esj.2018.v14n34p162

\begin{abstract}
The aim of this study consisted in determining the differences and similarities among Mexican high school students concerning their selfconcept. A total sample of 544 participants, 264 women and 280 men, with a mean age of 12.96 years ( $D S=0.94)$ and 12.85 years $(D S=0.98)$ respectively. A quantitative approach with a descriptive and transversal survey design was used. All the participants completed the Self-concept Questionnaire AUDIM of Rodríguez and Fernández (2011). The results of the one-way multivariate analysis of variance, followed by the one-way univariate analyses of variance, showed that compared with the women, the men obtained higher scores on the subscales Physical Self-concept, Social Self-concept and General Selfconcept. However, in Academic and Personal Self-concept subscales statistically significant differences were not found. Further research should reply these findings in larger samples.
\end{abstract}

Keywords: Self-concept, gender, student's beliefs, self-perceptions

\section{Resumen}

El objetivo de esta investigación consistió en determinar las diferencias y similitudes entre alumnos y alumnas de secundaria mexicanos en cuanto a la percepción sobre su autoconcepto. La muestra total fue de 544 sujetos; 264 mujeres y 280 hombres, con una edad media de 12.96 años (DE= $0.94)$ y 12.85 años $(\mathrm{DE}=0.98)$ respectivamente. El abordaje adoptado en la investigación se enmarcó dentro de un enfoque cuantitativo con un diseño descriptivo tipo encuesta. Todos los participantes contestaron el Cuestionario 
Autoconcepto AUDIM de Rodríguez y Fernández (2011). Los resultados del análisis multivariante de la varianza, seguido por los análisis de varianza univariados, muestran que los hombres en comparación con las mujeres son quienes obtuvieron puntuaciones más altas en las subescalas de Autoconcepto Físico, Autoconcepto Social y Autoconcepto General. Sin embargo, en las subescalas Autoconcepto Académico y Personal no se encontraron diferencias estadísticamente significativas. Futuras investigaciones deberían replicar estos hallazgos en muestras más amplias.

Palabras clave: Autoconcepto, género, creencias de estudiantes, autopercepciones

\section{Introducción:}

El autoconcepto es una de las construcciones más importantes en el campo de la investigación motivacional por ello la psicología siempre le ha prestado una atención preferencial; considerándolo como un factor predictivo importante del comportamiento y de los resultados emocionales y cognitivos de las personas (Marsh y Martin, 2011). El autoconcepto puede ser definido como las autopercepciones de la propia persona que se forman a través de la experiencia e interpretaciones del propio entorno (Shavelson, Hubner y Stanton, 1976).

El autoconcepto juega un papel central en el desarrollo de la personalidad; un autoconcepto positivo es la base de un buen funcionamiento personal, social y profesional, resultando ser un buen indicador de salud mental y de ajuste con la vida (A. Goñi, 2009; E. Goñi y Infante, 2010; Reigal, Videra, Parra y Juárez, 2012) puesto que el sentirnos a gusto con nosotros mismos ayuda a generar sentimientos positivos. De ahí que lograr un autoconcepto positivo sea uno de los objetivos más pretendidos en numerosos programas de intervención psicológica (Esnaola, Goñi y Madariaga, 2008).

Uno de los modelos multidimensionales más difundido y aceptado, entre los propuestos, es el de Shavelson et al. (1976) según el cual el autoconcepto general ocupa la parte superior de la jerarquía quedando dividido en autoconcepto académico y en autoconcepto no académico. El autoconcepto no académico comprende a su vez los dominios del autoconcepto social, emocional y físico (Marsh, 1987; Marsh y Shavelson, 1985). Modelo en el cual está basado nuestro trabajo.

La presente investigación es fundamentalmente un estudio de tipo descriptivo que intenta comparar los perfiles de autoconcepto de estudiantes de secundaria mexicanos. Esta investigación pretende aportar información que se traduzca en una práctica educativa de mayor calidad en el contexto de atención a la diversidad; contribuyendo al saber pedagógico en el esclarecimiento de los factores que conforman un modelo de desarrollo 
humano integral; bajo la premisa de que los esfuerzos educativos deben enfocarse hacia el aumento los sentimientos de autovaloración y competencia de los estudiantes, fortaleciendo su autoconcepto, lo que a su vez favorecerá la motivación hacia el logro, las relaciones interpersonales y en general la forma particular de desenvolverse frente a diversas tareas y desafíos que se les presenten.

\section{Método \\ Participantes}

Participaron en el estudio 544 sujetos, 264 mujeres y 280 hombres estudiantes de secundaria de la ciudad de Chihuahua, México; con una edad media de 12.96 años $(\mathrm{DE}=0.94)$ y 12.85 años $(\mathrm{DE}=0.98)$ respectivamente.

\section{Instrumento}

Cuestionario Autoconcepto AUDIM de Rodríguez y Fernández (2011), es una encuesta tipo Likert, asistida por computadora, de 31 ítems relacionados con la propia persona; donde el encuestado responde, en una escala del 1 a 5 (1= Falso, 2=Más bien falso 3=Ni verdadero ni falso 4=Más bien verdadero y $5=$ Verdadero) su grado de acuerdo con cada uno de los aspectos propuestos (escogiendo la respuesta que más se ajuste a su persona). Los ítems del cuestionario se agrupan en cuatro factores específicos: Autoconcepto Académico (6 ítems), Autoconcepto Social (4 ítems), Autoconcepto Físico (8 ítems) y Autoconcepto Personal (8 ítems) y uno general Autoconcepto General (5 ítems).

Para nuestro estudio se hizo la siguiente adaptación: mientras que en la escala original se puntúa con cinco opciones de respuesta, en la versión utilizada en la presente investigación, el sujeto elige entre once posibles opciones en una escala de 0 a 10, esta adaptación se justifica en relación a que los sujetos están acostumbrados a la escala de 0 a $10(0=$ Falso, 1 a 3=Casi siempre falso, 4 a $6=\mathrm{A}$ veces falso a veces verdadero, 7 a $9=$ Casi siempre verdadero y $10=$ Verdadero) (Figura 1 ), ya que así han sido evaluados por el sistema educativo de nuestro país (México). Viciana, Cervelló y Ramírez (2007) reportan un cambio similar en la validación de una escala con población española. 


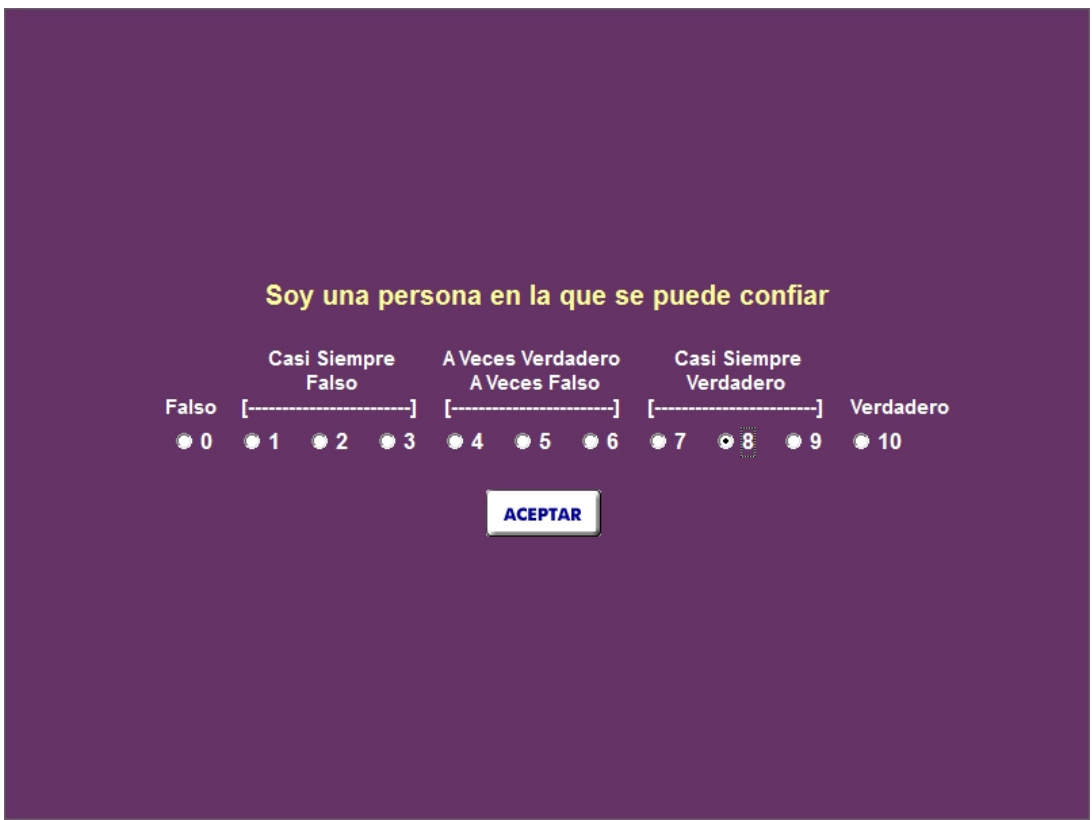

Figura 1. Ejemplo de respuesta para los ítems del cuestionario.

\section{Diseño}

En cuanto al diseño del estudio, se utilizó un enfoque cuantitativo con un diseño descriptivo y transversal tipo encuesta (Hernández, Fernández y Baptista, 2014). La variable independiente fue el Género y las variables dependientes el promedio de las puntuaciones obtenidas en cada uno de los factores del cuestionario de autoconcepto AUDIM.

\section{Procedimiento}

Una vez conseguido el permiso tanto de las autoridades educativas como el de las familias, se invitó a participar en el estudio a los alumnos y alumnas de secundaria de la ciudad de Chihuahua.

Los que aceptaron participar firmaron el consentimiento informado. Luego se aplicó el instrumento por medio de una computadora personal, en una sesión de aproximadamente 30 minutos; en las aulas de los centros educativos.

Al inicio de cada sesión se hizo una pequeña introducción sobre la importancia de la investigación y de cómo acceder al instrumento. Se les solicitó la máxima sinceridad y se les garantizó la confidencialidad de los datos que se obtuvieran. Las instrucciones de cómo responder se encontraban en las primeras pantallas; antes del primer reactivo del instrumento. Al término de la sesión se les agradeció su participación. 
Una vez aplicado el instrumento se procedió a recopilar los resultados por medio del módulo generador de resultados del editor de escalas versión 2.0 (H. Blanco et al., 2013).

\section{Análisis de datos}

Se realizaron análisis estadísticos descriptivos (medias y desviaciones estándar) para todas las variables. Posteriormente, después de verificar que los datos cumplían con los supuestos de los análisis estadísticos paramétricos, se llevó a cabo un análisis multivariante de varianza (MANOVA), seguido por los análisis de varianza univariados (ANOVAs), para examinar las diferencias entre hombres y mujeres en cuanto a la percepción de su autoconcepto en cada uno de los factores del AUDIM. Por otra parte, el tamaño del efecto se estimó mediante el eta cuadrado $\left(\eta^{2}\right)$. Todos los análisis estadísticos se realizaron con el programa SPSS versión 20.0 para Windows. El nivel de significación estadística se estableció en $\mathrm{p}=.05$.

\section{Resultados}

La Tabla 1 muestra los valores de las medias y las desviaciones estándar de las puntuaciones obtenidas en cada uno de los factores del cuestionario de autoconcepto AUDIM, así como los resultados del MANOVA y los subsiguientes ANOVAs.

Los resultados del MANOVA mostraron diferencias globales estadísticamente significativas de acuerdo a la variable género en las puntuaciones del autoconcepto (Wilks $\lambda=.899, \mathrm{p}<.001 ; \eta^{2}=.101$ ). Posteriormente, los ANOVAs indicaron que, en comparación con las adolescentes, los adolescentes muestran puntuaciones más altas en autoconcepto físico $\left(\mathrm{F}=39.782, \mathrm{p}<.001 ; \eta^{2}=.068\right)$, autoconcepto social $(\mathrm{F}=$ 9.097, $\left.\mathrm{p}<.01 ; \eta^{2}=.017\right)$ y autoconcepto general $\left(\mathrm{F}=31.916, \mathrm{p}<.001 ; \eta^{2}=\right.$ .056). En las subescalas de autoconcepto personal y académico no se encontraron diferencias significativas.

Tabla 1.

Resultados del MANOVA y ANOVAs para las diferencias en las puntuaciones obtenidas en cada uno de los factores del autoconcepto de acuerdo a la variable género

\begin{tabular}{cccccc}
\hline & $\begin{array}{c}\text { mujeres } \\
(n=264)\end{array}$ & $\begin{array}{c}\text { hombres } \\
(n=280)\end{array}$ & $F$ & $p$ & $\eta^{2}$ \\
\hline & & & 12.079 & $<.001$ & .101 \\
Autoconcepto Físico & $5.96(1.70)$ & $6.81(1.44)$ & 39.782 & $<.001$ & .068 \\
Autoconcepto Personal & $6.65(1.41)$ & $6.82(1.29)$ & 2.115 & $>.05$ & .000 \\
Autoconcepto Social & $6.64(1.91)$ & $7.08(1.48)$ & 9.097 & $<.01$ & .017 \\
Autoconcepto Académico & $6.64(1.96)$ & $6.61(1.91)$ & 0.040 & $>.05$ & .000 \\
Autoconcepto General & $6.52(2.02)$ & $7.42(1.67)$ & 31.916 & $<.001$ & .056 \\
\hline
\end{tabular}

Nota. Los valores descriptivos se presentan como media (desviación estándar) 


\section{Discusión y Conclusiones:}

Los resultados muestran que, aun cuando no se encontraron diferencias significativas entre las y los adolescentes en cuanto a su autoconcepto personal y académico, las mujeres muestran un autoconcepto más pobre en el resto de las dimensiones, incluida la de autoconcepto físico donde el tamaño del efecto es el más importante de los resultados que en general concuerdan con los obtenidos por J. R. Blanco, Rodríguez-Villalobos, Barrón y Medina (2015) solo que en una muestra de jóvenes universitarios mexicanos; de tal forma que las mujeres al manifestar niveles más bajos de autoconcepto físico presentan un mayor riesgo de padecer trastornos en la alimentación, ya que se ha comprobado que las personas con un pobre autoconcepto físico son más vulnerables a la presión cultural en pro de un cuerpo más delgado (A. Goñi y Rodríguez, 2004; E. Goñi y Infante, 2010; Rodríguez, González-Fernández y Goñi, 2013). Por otro lado, los autores Meza-Peña y Pompa-Guajardo (2015) a diferencia de la presente investigación encontraron que en el autoconcepto social, las mujeres fueron quienes reportaron mayores puntuaciones. No obstante, es preciso desarrollar más investigación al respecto pues el tema trasciende los alcances de este estudio.

Finalmente, cuando menos dos limitaciones están presentes en este trabajo. La primera es que los participantes son solo estudiantes de secundaria mexicanos, lo que supone una amenaza para la posibilidad de generalizar estos resultados. Ampliar la muestra (agregando por ejemplo adolescentes que no son estudiantes) es un área de trabajo de cara al futuro. La segunda limitación proviene del propio instrumento de evaluación, que se basa en el autoinforme y que puede contener los sesgos que se derivan de la deseabilidad social.

\section{Agradecimientos}

Este estudio es parte de un proyecto financiado por la Secretaría de Educación Pública-Subsecretaría de Educación Superior-Dirección de Superación Académica- Programa para el Desarrollo Profesional Docente (DE-13 -6894).

\section{References:}

1. Blanco, H., M. Ornelas, J. L. Tristán, A. Cocca, D. Mayorga-Vega, J. López-Walle y J. Viciana. 2013. Editor for creating and applying computerise surveys. Procedia Social and Behavioral Sciences, 106, 935-940. doi: 10.1016/j.sbspro.2013.12.105

2. Blanco, J. R., J. M. Rodríguez-Villalobos, J. C. Barrón y H. L. Medina. 2015. Self-Concept in Young Mexicans, a Comparison by Gender. American Journal of Applied Psychology, 4(6), 163-165. doi: 10.11648/j.ajap.20150406.16 
3. Esnaola, I., A. Goñi y J. M. Madariaga. 2008. El autoconcepto: perspectivas de investigación. Revista de Psicodidáctica, 13(1), 69-96.

4. Goñi, A. 2009. El autoconcepto físico: Psicología y educación. Madrid: Pirámide.

5. Goñi, A. y A. Rodríguez. 2004. Trastornos de conducta alimentaria, práctica deportiva y autoconcepto físico en adolescentes. Actas Españolas de Psiquiatría, 32(1), 29-36.

6. Goñi, E. y G. Infante. 2010. Actividad físico-deportiva, autoconcepto físico y satisfacción con la vida. European Journal of Education and Psychology, 3(2), 199-208.

7. Hernández, R., C. Fernández y P. Baptista. 2014. Metodología de la Investigación. México: McGraw- Hill.

8. Marsh, H. W. 1987. The hierarchical structure of the self-concept: An application of hierarchical confirmatory factor analysis. Journal of Educational Measurement, 24, 17-39.

9. Marsh, H. W. y A. J. Martin. 2011. Academic self-concept and academic achievement: Relations and causal ordering. British Journal of Educational Psychology, 81(1), 59-77.

10. Marsh, H. W. y R. J. Shavelson. 1985. Self-concept: Its multifaceted, hierarchical structure. Educational Psychologist, 20(3), 107-123.

11. Meza-Peña, C. y Pompa-Guajardo, E. G. (2015). Género, obesidad y autoconcepto en una muestra de adolescentes de México.[Gender, obesity and self concept in a sample of Mexican adolescents]. RICYDE. Revista Internacional de Ciencias del Deporte. doi: 10.5232/ricyde, 12(44), 137-148.

12. Reigal, R., A. Videra, J. L. Parra y R. Juárez. 2012. Actividad físico deportiva, autoconcepto físico y bienestar psicológico en la adolescencia. Retos. Nuevas tendencias en Educación Física, Deporte y Recreación, 22, 19-23.

13. Rodríguez, A. y A. Fernández. 2011. Propiedades psicométricas del Cuestionario de Autoconcepto Dimensional AUDIM. En J. M. Román, M. Á. Carbonero \& J. D. Valdivieso (Eds.), Educación, aprendizaje y desarrollo en una sociedad multicultural (pp. 999-1113). Madrid: Ediciones de la Asociación Nacional de Psicología y Educación.

14. Rodríguez, A., Ó. González-Fernández y A. Goñi. 2013. Sources of perceived sociocultural pressure on physical self-concept. Psicothema, 25(2), 192-198. doi: 10.7334/psicothema2012.229

15. Shavelson, R. J., J. J. Hubner y G. C. Stanton. 1976. Self concept: Validation of construct interpretations. Review of Educational Research, 46, 407-441.

16. Viciana, J., E. M. Cervelló y J. Ramírez. 2007. Effects of manipulating positive and negative feedback on goal orientation, perceived 
motivational climate, satisfaction, task choice, perception of ability, and attitude to physical education lessons. Perceptual and motor skills, 105(1), 67-82. 